Original paper

\title{
Allopurinol potentiates the hepatoprotective effect of metformin and vitamin $E$ in fructose-induced fatty liver in rats
}

\author{
Hanaa Mohamed Khalaf', Mohamed Abdellah Ibrahim¹, Entesar Farghaly Amin', Salwa Abdel-tawab Ibrahim', \\ Soha Abdel-Wahab'2 ${ }^{2}$ Yasser Mahrous Fouad ${ }^{3}$ \\ 'Department of Pharmacology, Faculty of Medicine, Minia University, Egypt \\ ${ }^{2}$ Department of Histology, Faculty of Medicine, Minia University, Egypt \\ ${ }^{3}$ Department of Tropical Medicine, Faculty of Medicine, Minia University, Egypt
}

\begin{abstract}
Aim of the study: Non-alcoholic fatty liver disease (NAFLD) is a challenging health problem. Hyperuricemia is a key player in the pathogenesis of NAFLD. This study investigated the effect of allopurinol (uric acid synthesis inhibitor) in combination with metformin and vitamin $\mathrm{E}$ in prevention of fructose induced-fatty liver in rats.

Material and methods: Rats were divided into 7 groups: control group, fructose group (model group of NAFLD), allopurinol-treated group, metformin-treated group, vitamin E-treated group, metformin plus vitamin E-treated group and a combination group (received allopurinol plus metformin plus vitamin E). Development of NAFLD was assessed biochemically by serum alanine aminotransferase (ALT) and aspartate aminotransferase (AST) as well as by histopathological examination. Oxidative stress parameters [reduced glutathione (GSH), superoxide dismutase (SOD), malondialdehyde (MDA)], and the inflammatory mediators tumor necrosis factor $\alpha$ (TNF- $\alpha$ ) and inducible nitric oxide synthase (iNOS) were assessed, along with serum levels of uric acid and triglyceride (TG).

Results: Combination of allopurinol plus metformin plus vitamin E significantly attenuated fatty changes compared to their respective monotherapy. Interestingly, though all treated groups showed significant attenuation in the oxidative stress markers, TNF- $\alpha$ level and iNOS immunostaining in hepatic tissue, along with a significant decrease in the levels of uric acid and $\mathrm{TG}$, the combination group showed a further significant decrease in the serum level of uric acid and iNOS immunostaining compared to other treated regimens.

Conclusions: Allopurinol synergistically increases the protective effect of metformin and vitamin $\mathrm{E}$ in treatment of NAFLD, namely via reduction of uric acid synthesis and iNOS expression.
\end{abstract}

Key words: allopurinol, NAFLD, metformin, vitamin E.

Address for correspondence

Mohamed Abdellah Ibrahim, MD, PhD, Assoc. Prof., Department of Pharmacology, Faculty of Medicine, Minia University, Minia, 61511, Egypt, phone: 00201023168222, fax: 0020862342813, e-mail: mabdellah69@mu.edu.eg, maim69@yahoo.com

\section{Introduction}

Non-alcoholic fatty liver disease (NAFLD) is an escalating and challenging global health problem. It has a complex pathogenesis pathway and lacks of satisfactory drug therapy. NAFLD may progress to non-alcoholic steatohepatitis (NASH), liver cirrhosis, and hepatocellular carcinoma. Nutritional factors, insulin resistance, oxidative stress, inflammation, and genetic factors are involved in the development of NAFLD [1-3].

A plethora of drugs such as insulin sensitizers, antioxidants, and lipid-lowering drugs have been tried as potential therapies for NAFLD, all without a satisfactory outcome [4]. Hence, optimizing the therapeutic strategy for NAFLD remains a medical challenge. 
Relying on the multisystem effects of NAFLD, combination therapy should be considered in planning the treatment strategy. Among the suggested promising drugs for treatment of NAFLD are metformin and vitamin E. Metformin, an insulin sensitizer, has been shown to decrease fat deposition and inhibit the inflammatory responses in hepatocytes [5]. The role of vitamin $\mathrm{E}$ in the treatment of NAFLD is based on its activity as an antioxidant [6].

A growing body of evidence has demonstrated the association between the serum level of uric acid and the presence of NAFLD [7]. Hence, the current study aimed to compare the effect of the triple combination between uric acid lowering agent, allopurinol, plus metformin and vitamin $\mathrm{E}$ versus their respective monotherapy or double therapy on prevention of NAFLD in rats.

\section{Material and methods}

\section{Animals}

The current study was conducted on male albino rats weighing 200-250 g. Rats were housed in metallic cages and allowed free access to tap water and standard rat chow diet for 2 weeks before the start of the experiment. The facilities were maintained at $24^{\circ} \mathrm{C} \pm 2^{\circ} \mathrm{C}$ with a 12-h dark: light cycle. Animals were treated according to the ARRIVE guidelines and in accordance with the U.K. Animals Act, 1986 and accompanying guidelines, and EU Directive 2010/63/EU for animal studies. The experiment was adherent to the ethical guidelines of the local ethical committee and approved by the board of the Faculty of Medicine, Minia University, Egypt.

\section{Drugs and chemicals}

Metformin and allopurinol were obtained as powders from Cid CO., Egypt. Vitamin E oil was obtained from El Hekma CO., Egypt. Metformin and allopurinol powders were dissolved in distilled water and freshly prepared before administration. Vitamin E oil was dispersed in distilled water.

\section{Induction of fatty liver}

Fatty liver was induced by adding $10 \%$ fructose to the drinking water for 4 consecutive weeks [8].

\section{Experimental design}

Rats were divided into 7 groups of 6 animals each: normal control that received vehicle; positive control model group that was given $10 \%$ fructose in drinking water and received no treatment; allopurinol group that was treated with allopurinol $50 \mathrm{mg} / \mathrm{kg} /$ day; metformin group that was treated with metformin $300 \mathrm{mg} / \mathrm{kg} /$ day; vitamin E group that was treated with vitamin E $100 \mathrm{mg} /$ $\mathrm{kg} /$ day; metformin plus vitamin E group that was cotreated with metformin $300 \mathrm{mg} / \mathrm{kg} /$ day plus vitamin $\mathrm{E}$ $100 \mathrm{mg} / \mathrm{kg} /$ day; and allopurinol plus metformin plus vitamin $\mathrm{E}$ group that was co-treated with allopurinol $30 \mathrm{mg} / \mathrm{kg} /$ day plus metformin $150 \mathrm{mg} / \mathrm{kg} /$ day plus vitamin E $100 \mathrm{mg} / \mathrm{kg} /$ day.

The tested drugs were given orally by a stomach tube for 4 weeks from the first day of the study.

\section{Sample collection and storage}

At the end of the experiment, rats were weighed then sacrificed under anesthesia by using urethane (125 mg/kg i.p.). Blood samples were withdrawn from the abdominal aorta and centrifuged (5000 rpm for 10 minutes) for sera collection. The collected sera were used for the measurement of biochemical parameters. The liver was rapidly excised, cleaned of blood by saline, and weighed. For each rat, one part from the right lobe of the liver was kept in $10 \%$ formalin for histopathological evaluation, parts were cut in $5 \mu \mathrm{m}$ thicknesses then deparaffinized and rehydrated for immunostaining and another part was accurately weighed and processed for biochemical measurements.

\section{Biochemical measurements}

\section{Measurement of triglycerides in the serum and hepatic tissue}

Triglycerides (TG) level in the serum was measured using an enzymatic colorimetric kit (catalogue NO: TG 119050) according to the manufacturer's instructions. The indicator was quinoneimine that formed from hydrogen peroxide, 4-aminoantipyrine and 4-chlorophenol under the catalytic influence of peroxidases [9].

Extraction of lipids from the liver tissue was done using the Folch method. Equal parts from the liver were homogenized in chloroform/methanol $(2: 1)$ ( $1 \mathrm{~g}$ in $20 \mathrm{ml}$ ). Then, the homogenate was centrifuged to recover the liquid phase. The solvent then washed by 0.2 volumes ( $4 \mathrm{ml}$ for $20 \mathrm{ml}$ ) of $0.9 \% \mathrm{NaCl}$ solution. After centrifugation for 10 seconds, the mixture was centrifuged again (2000 revolutions per minute) for separation of the two phases. The upper phase was removed and the lower chloroform phase containing lipids was used for measurement of TG using an enzymatic colorimetric kit (catalog number: 31400) according to the manufacturer's instructions [10]. 


\section{Serum activities of alanine aminotransferase and aspartate aminotransferase}

Serum levels of alanine aminotransferase (ALT) and aspartate aminotransferase (AST) were assessed using enzymatic kinetic kits according to the manual instructions (catalog NO: EZ016LQ and EZ012LQ, respectively).

\section{Determination of uric acid in serum}

The level of uric acid in the serum was evaluated using the enzymatic colorimetric kit (catalog NO: UA119090). In this method, uricase enzyme oxidized uric acid to allantoin and hydrogen peroxide. The formed hydrogen peroxide led to oxidative coupling of 3,5-dichloro-2-hydroxybenzene sulfonate (DHBS) and 4-aminoantipyrine, in the presence of peroxidase, forming a red colored quinoneimine dye. The intensity of the color was directly proportional to the concentration of uric acid in the sample, with maximum absorbance at $550 \mathrm{~nm}$.

\section{Oxidative stress parameters in hepatic tissue}

Hepatic tissue samples were homogenized in phosphate buffer solution, then the homogenate was centrifuged for $15 \mathrm{~min}$ and the supernatant was used for measurement of oxidative stress parameters: malondialdehyde (MDA), superoxide dismutase (SOD) and reduced glutathione (GSH).

Hepatic MDA content, a lipid peroxidation indicator, was measured chemically by the spectrophotometric method. This was based on the reaction between MDA and thiobarbituric acid as previously described elsewhere [11].

SOD activity was assessed chemically as previously measured by Marklund and Markuland (1974) [12]. The process was based on the fact that SOD inhibits the autoxidation of pyrogallol. One unit of SOD is generally defined as the amount of enzyme that inhibits the autoxidation of pyrogallol by $50 \%$. The activity of SOD was measured at $420 \mathrm{~nm}$.

Hepatic content of GSH was measured as previously described [13]. The protein in liver homogenate was precipitated with trichloroacetic acid and then centrifuged at $1000 \mathrm{rpm}$ for $5 \mathrm{~min}$. The reaction mixture containing $0.25 \mathrm{ml}$ of supernatant, $1 \mathrm{ml}$ of $0.2 \mathrm{M}$ Tris$\mathrm{HCl}$ (containing $1 \mathrm{mM}$ EDTA, pH 8.9) and $0.05 \mathrm{ml}$ of $10 \mathrm{mM}$ 5,5'-dithiobis-(2-nitrobenzoic acid) in absolute methanol was kept at room temperature for $5 \mathrm{~min}$, and the yellow color developed was monitored by a spectrophotometer at $412 \mathrm{~nm}$.

\section{Tumor necrosis factor $\alpha$ in hepatic tissue}

Tumor necrosis factor a (TNF- $\alpha$ ) was determined using an ELISA (Enzyme-Linked Immunosorbent Assay) kit (Sigma-Aldrich, St. Louis, MO, USA) (catalog NO RAB0480). Monoclonal anti-rat antibodies for TNF- $\alpha$ were precoated onto 96-well plates. Samples and standard were added to the wells followed by addition of biotinylated antibody to each well. Avidin-Biotin-Peroxidase Complex (ABC) was added and a substrate solution was used and produced a blue color that changed to yellow color after adding acidic stop solution. The density of the color was monitored at $450 \mathrm{~nm}$ immediately.

\section{Histopathological examination of hepatic tissue}

Liver tissue samples were fixed in formalin and embedded in paraffin. Four micron-thickness sections were taken, stained with hematoxylin and eosin stain and examined under a light microscope in a blind manner. The severity of liver steatosis was graded as $1,2,3$, and 4 according to the extent of fatty cells as follows: 1 when fatty cells $\leq 25 \%$; 2 when fatty cells $=$ $26-50 \%$; 3 when fatty cells $=51-75 \% ; 4$ when fatty cells $>75 \%$ of cells. Counting the number of inflammatory foci was used for scoring of the inflammation [14].

\section{Immunostaining of iNOS in hepatic tissue}

For evaluating the iNOS immunoexpression, $5 \mu \mathrm{m}$ thicknesses from the right lobe of the liver were cut, deparaffinized in xylene and rehydrated in graded alcohol. Endogenous peroxidase activity was blocked using $\mathrm{H}_{2} \mathrm{O}$ / methanol, followed by adding citrate buffer ( $\mathrm{pH}$ 6.0). Then, the sections were incubated at room temperature for 40 min with the anti-iNOS monoclonal anti-rat antibodies (Sigma-Aldrich, St. Louis, MO, USA).

Detection of the staining was done using UltraVision (Thermo Scientific) according to the manufacturer's instructions. Scoring for immunostaining of iNOS was done by counting the number of stained cells using 5 high power fields for each rat [15].

\section{Statistical analysis}

Results were expressed as means \pm standard error of mean (S.E.M.). One-way analysis of variance (ANOVA) followed by the Tukey-Kramer test was used to analyze the results for statistically significant difference. $P$ values were considered to be significant if less than 0.05. GraphPad Prism was used for statistical calculations (version 5.01 for Windows, GraphPad Software, San Diego California USA, www.graphpad.com). 


\section{Results}

\section{Body weight and liver index}

There was a significant elevation in liver index (liver weight/body weight) in the fructose model group as compared to the control group. Rats treated with allopurinol, metformin, vitamin E, metformin plus vitamin E and allopurinol plus vitamin E plus allopurinol showed a significant decline in the liver index compared to the model group (Table 1).

\section{Serum activities of alanine aminotransferase and aspartate aminotransferase}

Serum activities of ALT and AST were significantly elevated in the fructose control group as compared with the normal control group. Groups treated with allopurinol, metformin, vitamin E or metformin plus vitamin $\mathrm{E}$ showed a significant amelioration in serum ALT and AST activities compared to the fructose group. Rats treated with a combination of metformin plus vitamin E plus allopurinol showed a further significant improvement in ALT and AST activities compared to other treated groups (Table 1).

\section{Serum level of uric acid}

In the fructose control group, serum uric acid was significantly elevation compared to the control group. Groups treated with allopurinol, metformin and/or vitamin $\mathrm{E}$ showed a significant decline in serum uric acid compared to fructose group and other groups. Rats treated with the triple combination (allopurinol/ vitamin E/metformin) revealed a further significant decline in serum level of uric acid compared to either treated group (Table 1).

\section{Triglycerides in serum and hepatic tissue}

Serum and hepatic levels of TG were significantly elevation in the fructose control group as compared with the normal control group. Treatment with allopurinol, metformin, vitamin E and their combination showed a significant amelioration in serum and hepatic TG level compared to the fructose group (Tables 1 and 2).

\section{Oxidative stress parameters in hepatic tissue}

There was a significant reduction in hepatic tissue content of GSH and the activity of SOD in the fructose group compared to the control group. Treatment with allopurinol, metformin and/or vitamin E, and their combination with allopurinol significantly increased hepatic content of GSH and activity of SOD levels compared to the fructose group. Hepatic content of MDA significantly increased in the fructose group and significantly decreased it in rats treated with metformin and vitamin $\mathrm{E}$ and their combination with allopurinol (Table 2).

\section{Tumor necrosis factor $\alpha$ in hepatic tissue}

In the fructose control group, there was a significant increase in hepatic tissue content of TNF- $\alpha$ compared with the control group. Treatment with allopurinol, metformin and/or vitamin $\mathrm{E}$ and their combination with allopurinol significantly decreased hepatic TNF- $\alpha$ level as compared to the fructose group (Fig. 1).

Table 1. Effect of allopurinol, metformin, vitamin E and their combination on liver index, and serum levels of liver enzymes, triglycerides, and uric acid

\begin{tabular}{|c|c|c|c|c|c|}
\hline Group & $\begin{array}{l}\text { Liver index } \\
\text { Liver weight (mg)/body weight (g) }\end{array}$ & $\begin{array}{l}\text { Serum TG } \\
(\mathrm{mg} / \mathrm{dl})\end{array}$ & $\begin{array}{l}\text { ALT } \\
(\mathrm{U} / \mathrm{I})\end{array}$ & $\begin{array}{l}\text { AST } \\
(\mathrm{U} / \mathrm{I})\end{array}$ & $\begin{array}{l}\text { Uric acid } \\
\text { (mg/dl) }\end{array}$ \\
\hline Control & $2.28 \pm 0.19$ & $91.50 \pm 5.98$ & $25.02 \pm 2.87$ & $21.20 \pm 3.78$ & $3.00 \pm 0.36$ \\
\hline Fructose (F) & $4.86 \pm 0.34^{*}$ & $166.70 \pm 8.82^{*}$ & $68.28 \pm 3.26^{*}$ & $60.60 \pm 3.21^{*}$ & $7.41 \pm 0.40$ * \\
\hline F/Allopurinol (A) & $3.74 \pm 0.11^{\#}$ & $110.32 \pm 7.13^{\#}$ & $37.67 \pm 2.14^{\#}$ & $40.60 \pm 2.50^{\text {*\# }}$ & $3.33 \pm 0.35^{\#}$ \\
\hline F/Metformin (M) & $3.56 \pm 0.29^{\#}$ & $109.70 \pm 3.73^{\#}$ & $43.10 \pm 3.94$ *\# & $41.40 \pm 3.20^{\text {*\# }}$ & $5.50 \pm 0.45^{* \#}$ \\
\hline F/Vitamin E (E) & $3.94 \pm 0.19^{\#}$ & $94.00 \pm 7.07^{\#}$ & $43.92 \pm 3.97^{\text {*\# }}$ & $39.41 \pm 5.90^{\text {*\# }}$ & $4.72 \pm 0.25^{\text {*\# }}$ \\
\hline$F / M+E$ & $3.02 \pm 1.02^{\#}$ & $94.67 \pm 6.76^{\#}$ & $34.67 \pm 1.23^{\#}$ & $30.60 \pm 1.66^{\#}$ & $5.25 \pm 0.30^{* \#}$ \\
\hline$F / A+M+E$ & $3.66 \pm 0.21^{\#}$ & $79.17 \pm 7.08^{\#}$ & $25.47 \pm 3.63^{\# \$}$ & $22.61 \pm 1.25^{\text {\#5 }}$ & $3.50 \pm 0.42^{\# 5}$ \\
\hline
\end{tabular}

Data represent the mean \pm SEM $(n=6)$. Results were considered significant when $p<0.05$. * Significant difference from control group. \#Significant difference from fructose group. sSignificant difference from allopurinol, metformin, vitamin E, and metformin + vitamin E groups, TG - triglycerides, ALT - alanine aminotransferase, AST - aspartate aminotransferase 
Table 2. Effects of allopurinol, metformin, vitamin E and their combination on oxidative stress parameters and triglycerides in hepatic tissue

\begin{tabular}{lcccc}
\hline Group & $\begin{array}{c}\text { GSH } \\
\text { (mg/g tissue) }\end{array}$ & $\begin{array}{c}\text { SOD } \\
\text { (U/g tissue) }\end{array}$ & $\begin{array}{c}\text { MDA } \\
\text { (nmol/g tissue) }\end{array}$ & $\begin{array}{c}\text { TG } \\
\text { (mg/g tissue) }\end{array}$ \\
\hline Control & $129.7 \pm 1.14$ & $1539 \pm 32.62$ & $81.24 \pm 5.61$ & $89.77 \pm 7.85$ \\
\hline Fructose (F) & $107 \pm 1.70^{*}$ & $1224 \pm 69.01^{*}$ & $128.73 \pm 2.53^{*}$ & $213.70 \pm 10.33^{*}$ \\
\hline F/Allopurinol (A) & $119.3 \pm 2.77^{* \#}$ & $1481 \pm 19.18^{\#}$ & $101 \pm 1.46^{* \#}$ & $90.80 \pm 3.31^{\#}$ \\
\hline F/Metformin (M) & $128.9 \pm 1.44^{\#}$ & $1548 \pm 38.68^{\#}$ & $98.23 \pm 2.83^{* \#}$ & $105.90 \pm 4.34^{\#}$ \\
\hline F/Vitamin E (E) & $122.9 \pm 1.76^{\#}$ & $1502 \pm 42.13^{\#}$ & $101.44 \pm 3.87^{* \#}$ & $92.20 \pm 3.48^{\#}$ \\
\hline F/M + E & $122.5 \pm 1.67^{\#}$ & $1502 \pm 31.66^{\#}$ & $92.83 \pm 2.24^{\#}$ & $101.8 \pm 3.07^{\#}$ \\
\hline F/A + M + E & $129.8 \pm 1.30^{\#}$ & $1530 \pm 41.14^{\#}$ & $91.26 \pm 1.76^{\#}$ & $97.09 \pm 4.04^{\#}$ \\
\hline
\end{tabular}

Data represent the mean \pm SEM $(n=6)$. Results were considered significant when $p<0.05 .{ }^{*}$ Significant difference from control group. "Significant difference from fructose group. sSignificant difference from allopurinol, metformin, vitamin E, and metformin + vitamin E groups. GSH - glutathione, SOD - superoxide dismutase, MDA - malondialdehyde, $T G$ - triglycerides.

\section{Histopathology of hepatic tissue}

Histopathological examination (Fig. 2 and Table 3) revealed hepatic architecture in the normal control group (A). In the fructose control group (B), there was a significant increase in liver steatosis and inflammation as compared to normal control rats. Treatment with allopurinol (C), metformin (D), vitamin E (E) or metformin plus vitamin $\mathrm{E}$ (F) caused significant attenuation of steatosis compared to the fructose group. Rat treated with the combination of metformin plus vitamin E plus allopurinol (G) showed a significant improvement in liver steatosis and inflammatory foci as compared to either the fructose group or other treated groups (Fig. 2, Table 3).

\section{Immunostaining of iNOS in hepatic tissue}

Figure 3 shows a significant increase in iNOS immunostaining in the model group (panel B) compared to the normal control (panel A). Rats treated with allopurinol (panel C), metformin (panel D), vitamin $\mathrm{E}$ (panel E) and metformin plus vitamin E (panel F) revealed a significant decrease in iNOS immunostaining compared with the non-treated model group (panel B). The rats treated with the combination of allopurinol plus metformin plus vitamin E (panel G) showed a significant decrease in the immunostaining of iNOS compared to other treated groups.

\section{Discussion}

Hyperuricemia plays a crucial role in the development of NAFLD [7, 16]. The present study presents a new finding that triple therapy with allopurinol plus metformin plus vitamin E showed higher efficacy in prevention of fructose-induced fatty liver than either

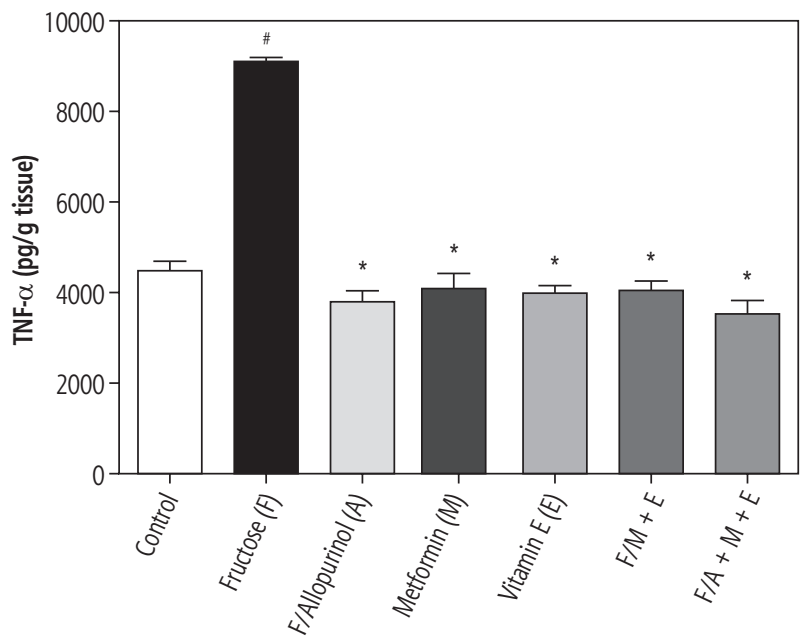

Fig. 1. Effects of allopurinol, metformin, vitamin $E$ and their combination with allopurinol on hepatic TNF- $\alpha$ level. Data represent the mean \pm SEM $(n=6)^{\#}$, *Significant difference from control group and fructose group, respectively $(p<0.05)$

the individual therapy or the double therapy with vitamin E plus metformin.

In this study, fructose-induced NAFLD was manifested by an elevation in the serum activities of liver enzymes (ALT and AST), indicating injury in liver cells. The damaged liver cells caused leakage of these cytoplasmic enzymes into the circulation $[17,18]$. Development of NAFLD was confirmed by histopathological examination in the form of microvesicular steatosis and inflammation accompanied with an increased liver index $[17,19]$.

The study elucidated the effect of the tested drugs on the pathogenesis of fructose-induced fatty liver by measuring metabolic and oxidative stress and inflammatory parameters.

In this study, fructose fed rats (model group) revealed an increase in serum and hepatic levels of TG along with a high serum level of uric acid $[20,21]$. 

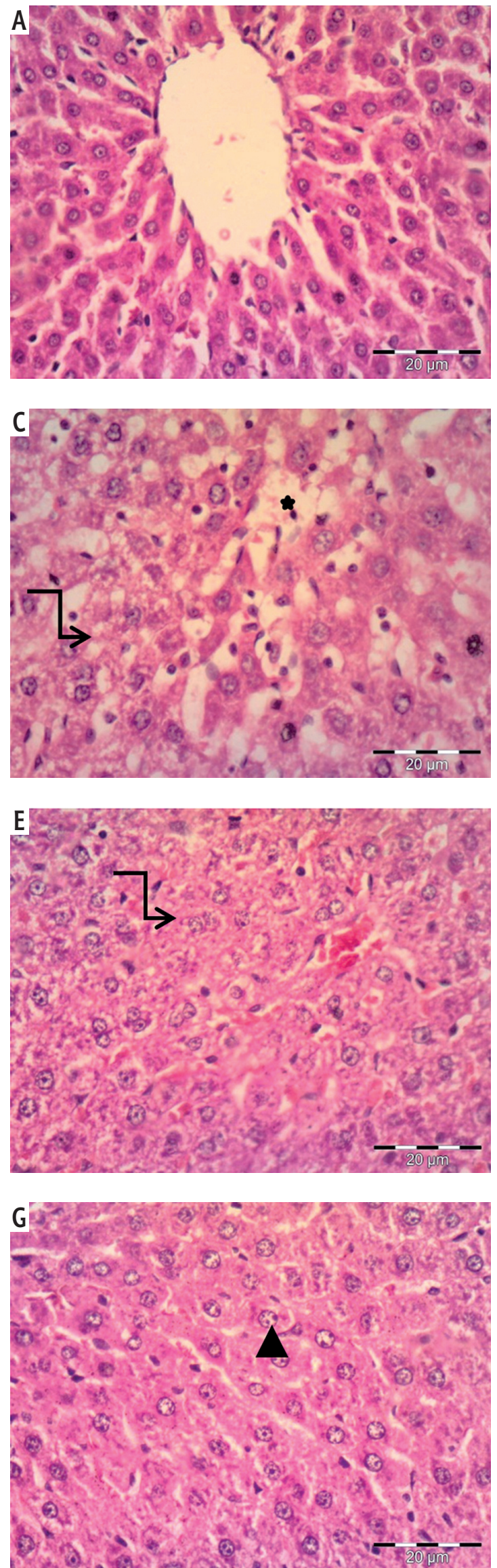
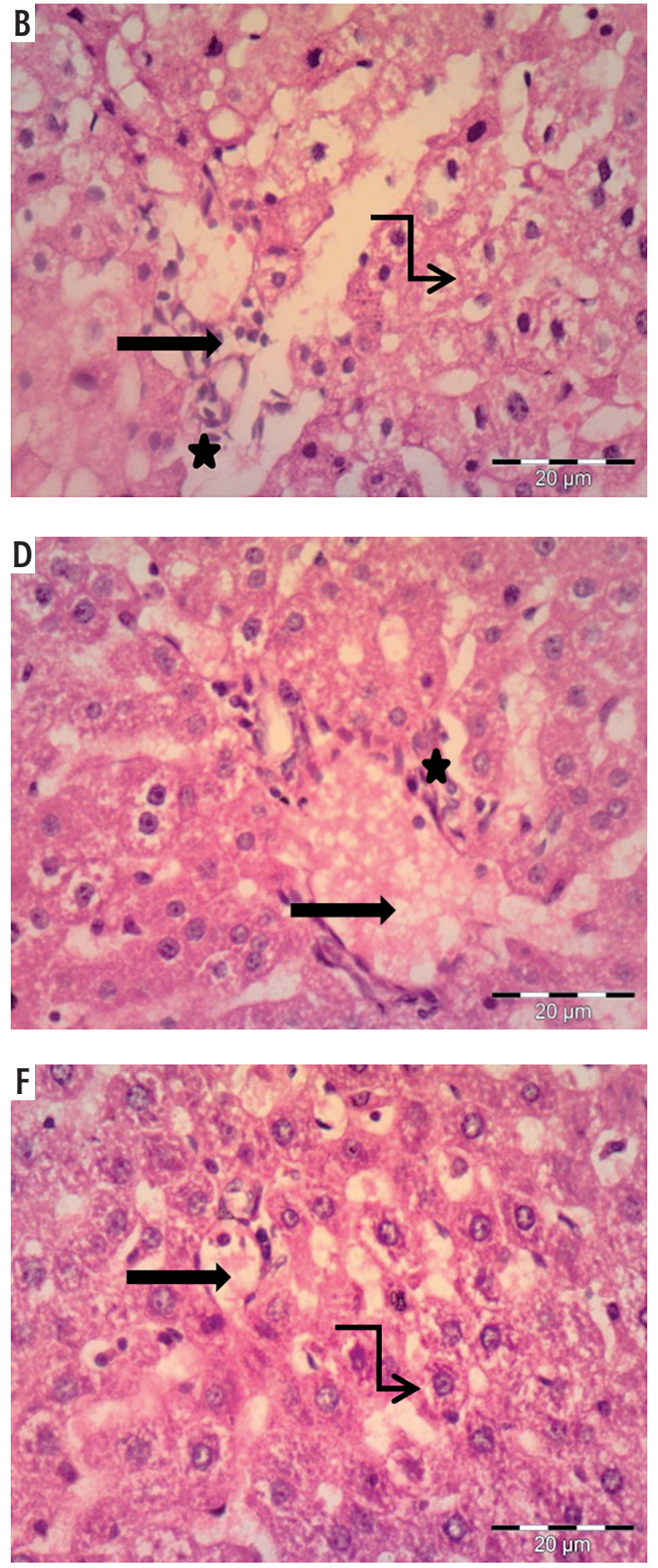

Fig. 2. Photomicrograph of the liver tissue: A) control group with normal liver histology, B) fructose group showing ballooning of hepatocytes with vacuolated cytoplasm and darkly stained nuclei (curved arrow), microvesicular steatosis (straight arrow), and inflammatory cell infiltration (star). C-E) Allopurinol, metformin and vitamin E treated groups, respectively showing mild $(\mathrm{D}, \mathrm{E})$ to moderate $(\mathrm{F})$ improvement in liver histology, $\mathrm{F})$ metformin plus vitamin $\mathrm{E}$ treated group showing moderate improvement in liver histology and G) combination group (allopurinol plus metformin plus vitamin E) showing significant improvement liver histology (most of the hepatocytes are back to normal (arrowhead). Note absence of inflammatory cell infiltration ( $\mathrm{H} \& \mathrm{E}$, scale bar $20 \mu \mathrm{m})$ 
Several explanations have been suggested for the association between TG and fructose-induced fatty liver. First, fructose is a lipogenic factor that leads to generation of TG as a direct product of its metabolism [22]. Second, chronic consumption of high fructose stimulates the secretion of apolipoprotein B48 with subsequent stimulation of intestinal synthesis of free cholesterol, and TG. Third, fructose, unlike glucose, does not enhance the release of insulin and leptin, which are considered the main factors that regulate energy production and adiposity [22].

Hyperuricemia is crucial in the development of fructose-induced fatty liver, and there is increasing evidence indicating that an elevated uric acid level represents a trigger for NAFLD development [23]. In the liver, ketohexokinase enzyme metabolizes fructose in a process that utilizes ATP to phosphorylate fructose to fructose-1-phosphate. Ketohexokinase enzyme, in contrast to hexokinases, which metabolize glucose, cannot cause a negative feedback effect. Consequently, there is no prevention of excessive phosphorylation with subsequent depletion of phosphate concentration inside the cell. Such an effect leads to stimulation of adenosine monophosphate deaminase, which converts adenosine monophosphate (AMP) to inosine monophosphate and eventually produces uric acid [23]. Hyperuricemia stimulates lipogenesis through induction of insulin resistance via direct inhibition of insulin receptor substrates. Additionally, hyperuricemia induces the inflammation and oxidative stress in liver cells [2, 24].

The present manuscript focused on the effect of allopurinol as an additional drug to the currently partially effective drugs (metformin and vitamin E).

Interestingly, the current results showed that rats treated with a combination of allopurinol plus metformin plus vitamin E caused a significant attenuation of NAFLD compared with either individual drug or double therapy (metformin plus vitamin E). This observation was evident in the histopathological examination as well as in the activities of liver enzymes. Such an effect was associated with significant attenuation of the serum level of uric acid. The hepatoprotective effects of allopurinol, vitamin $\mathrm{E}$ and metformin in NAFLD were reported in previous studies [25, 26].

As regard the signaling mechanisms, it has been reported that the stimulant impact of fructose on carbohydrate-responsive element-binding protein (ChREBP) was inhibited by using allopurinol [27]. The mechanism by which allopurinol blocks ChREBP nuclear translocation could be mediated by way of inactivation of AMP kinase, a recognized inhibitor of ChREBP activity [28]. Additionally, it has been reported that hyperuricemia induces insulin resistance
Table 3. Effect of allopurinol, metformin, vitamin $E$ and their combination on histopathological score of hepatic tissue

\begin{tabular}{lcc}
\hline Group & Steatosis & Inflammation \\
\hline Control & $0.00 \pm 0.00$ & $0.00 \pm 0.00$ \\
\hline Fructose (F) & $3.0 \pm 0.00^{*}$ & $1.66 \pm 0.33^{*}$ \\
\hline F/Allopurinol (A) & $1.45 \pm 0.01^{\text {*\# }}$ & $1.45 \pm 0.01^{*}$ \\
\hline F/Metformin (M) & $1.66 \pm 0.32^{\text {*\# }}$ & $1.00 \pm 0.00^{*}$ \\
\hline F/Vitamin E (E) & $2.33 \pm 0.31^{\text {*\# }}$ & $0.66 \pm 0.33^{*}$ \\
\hline F/M + E & $1.67 \pm 0.12^{\text {*\# }}$ & $1.012 \pm 0.04^{*}$ \\
\hline F/A + M + E & $0.66 \pm 0.30^{\# s}$ & $0.33 \pm 0.33^{\#}$ \\
\hline
\end{tabular}

Data represent the mean \pm SEM $(n=6)$. Results were considered significant when $p<0.05$. *Significant difference from control group. "Significant difference from fructose group. ssignificant difference from allopurinol, metformin, vitamin $E$, and metformin + vitamin $E$ groups.

(a principle pathway of NAFLD) via inhibition of phospho-insulin receptor substrate 1 (IRS1; Ser307), phospho-AKT (Ser473) signals and insulin-stimulated glucose uptake. Inhibition of uric acid synthesis reverses these signals, thereby preventing insulin resistance [29].

In NAFLD, oxidative stress is considered a key factor in hepatocellular injury. The mitochondrial respiratory chain is the main subcellular supply of reactive oxygen species, which may harm the mitochondrial proteins, lipids and mitochondrial DNA [30, 31]. In the current study, rats treated with allopurinol, metformin and/or vitamin $\mathrm{E}$, and metformin plus vitamin E plus allopurinol showed a decrease in the level of MDA and improved GSH and SOD enzyme activities. A previous study was carried out on a rat model of diabetes where metformin was noted to ameliorate mitochondrial oxidative stress as manifested by a reduction in mitochondrial aconitase activity [32]. Vitamin $\mathrm{E}$ is a lipid-soluble antioxidant that is incorporated into cell membranes, protecting them from oxidative damage. It acts as a chain-breaking antioxidant and repairs oxidizing radicals directly by preventing the chain propagation step $[33,34]$.

The data of the present study, consistently with previous studies, showed a significant increase in TNF- $\alpha$ in NAFLD $[35,36]$. This might be attributed to the ability of adipose tissue, an endocrine tissue, to produce some of the proteins termed adipokines, which include leptin, TNFa, interleukin (IL)-6, resistin, and adiponectin. Such substances play an important role in the inflammatory process and insulin resistance associated with NAFLD [36].

In the current study, treatment of rats with allopurinol, metformin and/or vitamin $\mathrm{E}$ attenuated fructose-induced liver injury as indicated by the improve- 

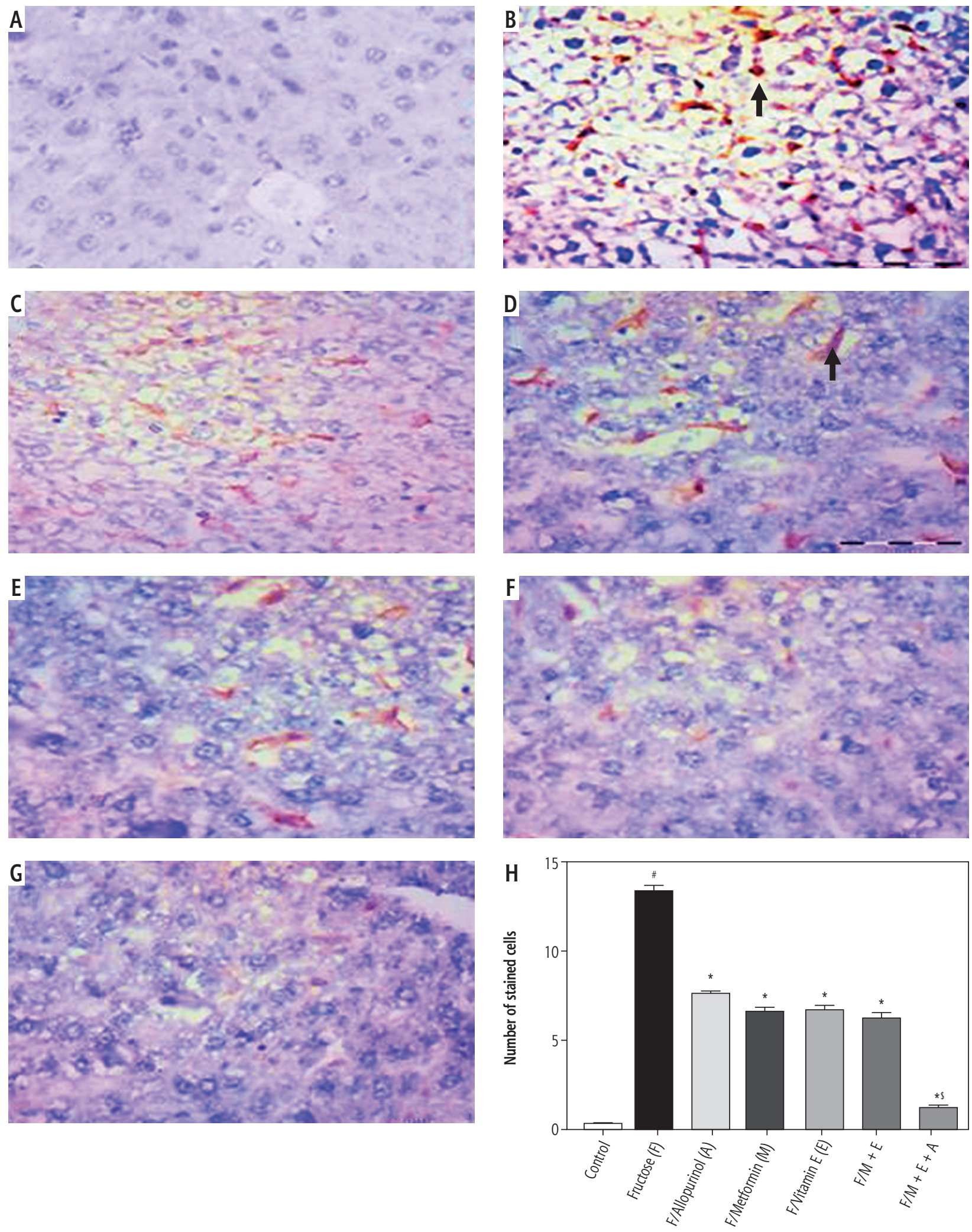

Fig. 3. iNOS immunostaining: A) negative expression of iNOS in liver tissue of (B) positive iNOS immunostaining in numerous cells (arrow) of liver tissue of fructose treated group. Note that the expression is mainly in nonparenchymal cells. C-E) panels show a decrease in the number of immunopositive cells in allopurinol, metformin and vitamin E treated groups, respectively. Note: low intensity of immunostaining. F) shows very few positive cells in metformin plus vitamin E treated group. G) Panel shows nearly negative expression of (INOS) in liver tissue of combination group (allopurinol plus metformin plus vitamin E); hematoxylin counterstain, scale bar $20 \mu \mathrm{m}$. $\mathbf{H}$ represents the score of iNOS expression (number of positive stained cells) in hepatic tissue. Data are presented as mean \pm SEM. \#, ${ }^{*}$, significant compared to normal control, fructose non-treated, and either monotherapy or double therapy treated groups, respectively 
ment in the activity of liver enzymes (ALT and AST) and confirmed by histopathological examination of liver tissue as well as by a reduction in the liver index. Additionally, the tested drugs reduced serum levels of uric acid and hepatic tissue content of TG, along with improvement of oxidative stress parameters. These findings were in consistence with previous studies [37-40]. It has been reported that hyperuricemia and oxidative stress might release the inflammatory mediators including TNF- $\alpha$ and iNOS that participate in the pathogenesis of NAFLD-associated liver injury $[39,40]$.

The results of this study indicated that the tested drugs either alone or in combination mitigated the expression of iNOS in hepatic tissue. It is worth noting that the triple therapy with allopurinol plus vitamin $\mathrm{E}$ plus metformin revealed significant mitigation of iNOS expression as compared to either the individual or the double therapy regimen. The released iNOS leads to synthesis of a large quantity of $\mathrm{NO}$, eventually with an increase in the oxidative product peroxynitrite. Inhibition of uric acid synthesis can inhibit overexpression of iNOS, leading to amelioration of excessive production of the end product NO. These effects were observed in the present study and in previous studies [16].

\section{Conclusions}

In summary, metformin, and vitamin $\mathrm{E}$ (either individually or in combination) showed a kind of protection against fructose-induced NAFLD. Their effects were associated with a reduction in oxidative stress parameters, TG, and the expression of TNF- $\alpha$ and iNOS in hepatic tissue. However, addition of allopurinol to vitamin $\mathrm{E}$ and metformin (i.e. triple therapy) caused a synergistic effect in prevention of fructose-induced NAFLD that was evident biochemically by liver enzymes (ALT and AST) and ascertained by histopathological examination. Such a synergistic effect was accompanied with a further significant reduction in the serum level of uric acid and hepatic iNOS immunostaining compared with other treatment regimens. The current results imply that the combination therapy between allopurinol plus metformin plus vitamin $\mathrm{E}$ is better in prevention of NAFLD than either individual or double therapy. It is worth noting that further large scale animal and human studies are needed to confirm the present findings.

\section{Acknowledgements}

This study did not receive any funds from any funding body. The study was funded by the Faculty of Medicine, Minia University, Egypt.

\section{Disclosure}

Authors report no conflict of interest.

\section{References}

1. Buzzetti E, Pinzani M, Tsochatzis EA. The multiple hit pathogenesis of non-alcoholic fatty liver disease (NAFLD). Metabolism 2016; 65: 1038-4865.

2. Derra A, Bator M, Menżyk T, et al. Underrated enemy - from nonalcoholic fatty liver disease to cancers of the gastrointestinal tract. Clin Exp Hepatol 2018; 4: 55-71.

3. Takahashi Y, Sugimoto K, Inui H, et al. Current pharmacological therapies for nonalcoholic fatty liver disease/nonalcoholic steatohepatitis. World J Gastroenterol 2015; 21: 3777-3785.

4. Linden MA, Lopez KT, Fletcher JA, et al. Combining metformin therapy with caloric restriction for the management of type 2 diabetes and nonalcoholic fatty liver disease in obese rats. Appl Physiol Nutr Metab 2015; 40: 1038-1047.

5. Song YM, Lee YH, Kim JW, et al. Metform in alleviates hepatosteatosis by restoring SIRT1-mediated autophagy induction via an AMP-activated protein kinase-independent pathway. Autophagy 2015; 11: 46-59.

6. Filozof C, Goldstein BJ, Williams RN, et al. Non-alcoholic steatohepatitis: limited available treatment options, but promising drugs in development and recent progress towards a regulatory approval pathway. Drugs 2015; 75: 1373-1392.

7. Yuan H, Yu C, Li X, et al. Serum uric acid levels and risk of metabolic syndrome: a dose-response meta-analysis of prospective studies. J Clin Endocrinol Metab 2015; 100: 4198-4207.

8. Choi YJ, Shin HS, Choi HS, et al. Uric acid induces fat accumulation via generation of endoplasmic reticulum stress and SREBP1c activation in hepatocytes. Lab Invest 2014; 94: 1114-1125.

9. Schettler G, Nüssel E. Recent results of the Hiedelberg research into the epidemiology of cardiac infarction. Dtsch Med Wochenschr 1975; 99: 2003-2008.

10. Folch J, Lees M, Stanley GHS. A simple method for the isolation and purification of total lipids from animal tissues. J Biol Chem 1957; 226: 497-509.

11. Buege JA, Aust SD. Microsomal lipid peroxidation. Methods Enzymol 1978; 52: 302-310.

12. Marklund S, Marklund G. Involvement of the superoxide anion radical in the autoxidation of pyrogallol and a convenient assay for superoxide dismutase. Eur J Biochem 1974; 47: 469-474.

13. Moron MS, Depierre JW, Mannervik B. Levels of glutathione, glutathione reductase and glutathione S-transferase activities in rat lung and liver. Biochim Biophys Acta 1979; 582: 67-78.

14. Ibrahim M, Farghaly E, Ibrahim S, et al. Montelukast and irbesartan ameliorate metabolic and hepatic disorders in fructose-inducedmetabolic syndrome in rats. Eur J Pharmacol 2014; 724: 204-210.

15. Ibrahim M, Farghaly E, Gomaa W, et al. Nitro-aspirin is a potential therapy for non-alcoholic fatty liver disease. Eur J Pharmacol 2011; 65: 289-295.

16. Hafez HM, Ibrahim MA, Ibrahim SA, et al. Potential protective effect of etanercept and aminoguanidine in methotrexate-induced hepatotoxicity and nephrotoxicity in rats. Eur J Pharmacol 2015; 768: 1-12.

17. Chung M, Ma J, Patel K, et al. Fructose, high-fructose corn syrup, sucrose, and nonalcoholic fatty liver disease or indexes of liver health: a systematic review and meta-analysis. Am J Clin Nutr 2014; 100: 833-849. 
18. Hadi NR, Al-Amran FG, Swadi A. Metformin ameliorates methotrexate- induced hepatotoxicity. J Pharmacol Pharmacother 2012; 3: 248-253.

19. de Castro UG, dos Santos RA, Silva ME, et al. Age-dependent effect of high-fructose and high-fat diets on lipid metabolism and lipid accumulation in liver and kidney of rats. Lipids in Health and Disease 2013; 12: 136.

20. Kostogrys RB, Pisulewski PM. Effect of conjugated linoleic acid (CLA) on lipid profile and liver histology in laboratory rats fed high-fructose diet. Environ Toxicol Pharmacol 2010; 30: 245-250.

21. Lanaspa MA, Sanchez-Lozada LG, Cicerchi C, et al. Uric acid stimulates fructokinase and accelerates fructose metabolism in the development of fatty liver. PLoS One 2012; 7: e47948.

22. Basciano H, Federico L, Adeli K. Fructose, insulin resistance, and metabolic dyslipidemia. Nutr Metab (Lond) 2005; 2: 5.

23. Darmawan G, Hamijoyo L, Hasan I. Association between serum uric acid and non-alcoholic fatty liver disease: a meta-analysis. Acta Med Indones 2017; 49: 136-147.

24. Barskova VG, Eliseev MS, Kudaeva FM, et al. Effect of metformin on the clinical course of gout and insulin resistance. Klin Med (Mosk) 2009; 87: 41-46.

25. Wang W, Wang C, Ding XQ, et al. Quercetinand allopurinol reduce liver thioredoxin-interacting protein to alleviate inflammation and lipid accumulation in diabetic rats. Br J Pharmacol 2013; 169: 1352-1371.

26. Khodaeian M, Tabatabaei-Malazy O, Qorbani M, et al. Effect of vitamins $\mathrm{C}$ and $\mathrm{E}$ on insulin resistance in diabetes: a meta-analysis study. Eur J Clin Invest 2015; 4: 1161-1174.

27. Li L, Zhang GF, Lee K, et al. A Western diet induced NAFLD in LDLR (-/)(-) mice is associated with reduced hepatic glutathione synthesis. Free Radic Biol Med 2016; 96: 13-21.

28. Foretz M, Ancellin N, Andreelli F, et al. Short-term overexpression of a constitutively active form of AMP-activated protein kinase in the liver leads to mild hypoglycemia and fatty liver. Diabetes 2005; 54: 1331-1339.

29. Yuan H, Hu Y, Zhu Y, et al. Metformin ameliorates high uric acid-induced insulin resistance in skeletal muscle cells. Mol Cell Endocrinol 2017; 443: 138-145.

30. Kehrer JP, Klotz LO. Free radicals and related reactive species as mediators of tissue injury and disease: implications for Health. Crit Rev Toxicol 2015; 45: 765-798.

31. Paradies G, Paradies V, Ruggiero FM, et al. Oxidative stress, cardiolipin and mitochondrial dysfunction in nonalcoholic fatty liver disease. World J Gastroenterol 2014; 20: 14205-14218.

32. Rosen P, Wiernsperger NF. Metformin delays the manifestation of diabetes and vascular dysfunction in Goto-Kakizaki rats by reduction of mitochondrial oxidative stress. Diabetes/Metabolism Research Reviews 2006; 22: 323-330.

33. Traber M, Atkinson J. Vitamin E, antioxidant and nothing more. Free Radic Biol Med 2007; 43: 4-15.

34. Jiang Q. Natural forms of vitamin E: metabolism, antioxidant, and anti-inflammatory activities and their role in disease prevention and therapy. Free Radic Biol Med 2014; 72: 76-90.

35. Stojsavljević S, Gomerčić Palčić M, Virović Jukić L, et al. Adipokines and proinflammatory cytokines, the key mediators in the pathogenesis of nonalcoholic fatty liver disease. World J Gastroenterol 2014; 20: 18070-18091.

36. Lin X, Zhang Z, Chen JM, et al. Role of APN and TNF- $\alpha$ in type 2 diabetes mellitus complicated by nonalcoholic fatty liver disease. Genet Mol Res 2015; 14: 2940-2946.

37. Souza-Mello V, Gregório BM, Cardoso-de-Lemos FS, et al. Comparative effects of telmisartan, sitagliptin and metformin alone or in combination on obesity, insulin resistance, and liver and pancreas remodelling in C57BL/6 mice fed on a very highfat diet. Clin Sci (Lond) 2010; 119: 239-250.

38. Demirel U, Yalniz M, Aygün C, et al. Allopurinol ameliorates thioacetamide-induced acute liver failure by regulating cellular redox-sensitive transcription factors in rats. Inflammation 2012; 35: 1549-1557.

39. Lima WG, Martins-Santos ME, Chaves VE. Uric acid as a modulator of glucose and lipid metabolism. Biochimie 2015; 116: 17-23.

40. Morsy MA, Ibrahim MA, Abd-Elghany MI. Dimethyl dimethoxy biphenyl dicarboxylate attenuates hepatic and metabolic alterations in high fructose-fed rats. Toxicol Ind Health 2016; 32: 59-67. 